INVESTIGACIONES

\title{
CONSTRUCCION DE PERFILES PSICOGRAFICOS DE PROFESORES DE EDUCACION BASICA DE ESCUELAS MUNICIPALES DE ARICA
}

\author{
Construction of psychographics profiles of teachers of basic education \\ of municipal schools of Arica
}

Alejandro Cuadra Peralta, Sau-lyn Lee Maturana, Pamela Sossa Roa

Universidad de Tarapacá, Departamento de Filosofía y Psicología, Avenida 18 de Septiembre 2222 Arica, Chile.

acuadra@uta.cl

\section{Resumen}

Se realizó un estudio descriptivo exploratorio para construir perfiles psicográficos de profesores de educación básica de Arica. El objetivo era explorar, a partir de las variables Actividades, Intereses y Opiniones, si existían características comunes entre los docentes. La investigación se llevó a cabo en dos fases: una cualitativa que incluye la recopilación de información a través de entrevistas individuales y grupales. $\mathrm{Y}$ otra cuantitativa que incluye la construcción de un Inventario Psicográfico, su validación y la administración de la Escala Maslach con el objetivo de encontrar alguna relación entre el síndrome de Burnout y los perfiles psicográficos de los profesores. Se encontraron cinco perfiles entre los cuales existen diferencias significativas entre ellos y el Burnout.

Palabras clave: educación municipal, formación docente, perfiles psicográficos, síndrome de Burnout.

\begin{abstract}
The purpose of this research was the realization of an exploratory descriptive study to build psychographics profiles of the primary education's teachers of Arica, to be able to distinguish the characteristics of the teachers, from the Activities, Interests and Opinions variables. The investigation concern two phases: a qualitative one that includes the recopilation of information (groupal and individual interviews). And another quantitative one that includes the construction of a Psychographics Inventory, the validation of the instrument and the application of the Maslach Burnout Inventory, to explore if there is any relation between Burnout syndrome and the teacher's psychographics profiles. We found five profiles which are significantly different among them and between the Burnout.
\end{abstract}

Key words: municipal education, teacher's formation, psychographics profiles, and Burnout syndrome. 


\section{INTRODUCCION}

La docencia en general supone profesionales comprometidos en la formación de sus estudiantes que se involucran, como personas en esa tarea, con todas sus capacidades y valores. De otra manera no lograrían la interrelación empática con sus alumnos, aspecto insustituible en la tarea docente (MINEDUC 2003).

Este importante rol que ejerce el profesor sobre el estudiante incluso ha determinado la elección profesional de sus educandos, en el sentido de que ellos mismos han declarado que cualidades tales como dedicación, compromiso, cuidado y devoción generosa, que han admirado en sus maestros cuando ellos eran niños durante la educación básica, posteriormente y durante su vida adulta han tratado de emularlas eligiendo la carrera de Pedagogía como elección profesional (June, Mohammed y Quamina 2001).

Es por eso que, en la actualidad, el Ministerio de Educación está preocupado por el ejercicio educativo y ha implementado una Evaluación del Desempeño Profesional Docente que busca el desarrollo y fortalecimiento sostenido de dicha profesión, específicamente del desempeño profesional, potenciando los procesos pedagógicos mediante el reconocimiento de sus fortalezas y el mejoramiento de sus debilidades, con el fin de favorecer mejores aprendizajes de los niños, niñas y jóvenes. En este sentido, el sistema de evaluación tiene un carácter fundamentalmente formativo (MINEDUC 2003). Por lo tanto, dicho Ministerio sostiene que un adecuado desempeño se puede lograr principalmente por medio de la formación teórica, del perfeccionamiento universitario, de la participación en seminarios académicos o por medio de pasantías al extranjero. Desde luego esto es importante; sin embargo, ha sido demostrado a través de investigaciones de percepción social de la Psicología Social, tales como el Efecto Pigmalión, y la prevalencia de daños y factores de riesgos de la salud física y mental de los profesores, confirmado en el Informe Estudio de Salud Laboral de los Profesores en Chile (Colegio de Profesores de Chile A. G. 2004), se supone pertinente que también se deben tomar en cuenta los aspectos psicológicos de los trabajadores, en este específico caso los profesores.

Es así que el sistema evalúa al docente exclusivamente por su ejercicio profesional, considerando el contexto y las condiciones de trabajo en que éste se desenvuelve. Pero ¿qué pasa con los aspectos psicológicos? y ¿cómo podríamos identificarlos?

Ante este planteamiento se considera pertinente indagar en la investigación de mercado, ya que posee una metodología que agrupa a las personas de acuerdo a sus características comunes. Esta caracterización recibe el nombre de Segmentación de Mercado y considera las variables o aspectos psicológicos que refieren a las Actividades, Intereses, Opiniones (AIO), motivaciones y valores para determinar perfiles psicográficos, los cuales describen las características y respuestas psicológicas de un individuo ante su medio ambiente (Klainer 2000).

Por otro lado y como bien lo describen Moriana y Herruzo (2004) en su estudio Estrés y Burnout en Profesores, el síndrome de Burnout o desgaste profesional ha estado presente en estos últimos años en el cuerpo docente. Es por esto que también surge la pregunta: ¿Habría alguna relación entre el síndrome de Burnout o desgaste profesional con las variables AIO anteriormente mencionadas? Y si así fuera, ¿de qué forma se podría manifestar?

Por lo anterior es que se considera que la formación del profesor es un proceso que parte desde las actividades, intereses y opiniones que emergen en la infancia y su vida 
estudiantil que motivaron su preferencia por el ejercicio docente, incluyendo su quehacer profesional actual hasta su futuro retiro laboral.

Frente al problema planteado se cree que es necesario realizar un estudio descriptivo exploratorio para construir perfiles psicográficos de los profesores de educación básica de escuelas municipales de Arica, lo que permitirá determinar las características de los docentes, a partir de las variables Actividades, de Intereses y de Opiniones asociadas a su quehacer profesional, así como también relacionar dichas características con el síndrome de Burnout que inicialmente se asociaba sólo a profesionales del área de la salud y que actualmente se ha ampliado a otras áreas como, por ejemplo, la docencia (Moriana y Herruzo 2004).

A continuación se describen los principales conceptos que atañen a la presente investigación a modo de contextualizar el estudio a la realidad local.

EDUCACION MUNICIPAL. A principios de la década de los 80 nace en Chile la Educación Municipal como una iniciativa del Estado con el fin de "descentralizar" la educación. Esto consiste en traspasar los recursos y la toma de decisiones a los municipios, logrando así una mayor autonomía en la gestión (Truffello 1999).

En Arica, el Servicio Municipal de Educación (SERME) fue creado el $1^{\circ}$ de agosto de 1980 durante el periodo del señor Alcalde de la época don Manuel Castillo Ibaceta. Desde entonces el SERME ha tenido como misión "Asegurar a la comunidad de Arica una educación de calidad que permita a sus alumnos tener acceso a la educación superior y/o vida laboral para enfrentar los desafíos que presenta el siglo XXI, manteniendo y acrecentando nuestro patrimonio cultural" (www.serme.cl).

Actualmente, el SERME, como organismo municipal, tiene entre sus principales compromisos: asegurar, administrar y proveer los recursos necesarios para que las unidades educativas puedan desarrollar con éxito sus propuestas; comprometer la participación activa de la comunidad en la educación; contar con los establecimientos educacionales cómodos, modernos, seguros y con equipamiento adecuado; y mantener capacitados permanentemente a los profesionales docentes para brindar una educación moderna (www.serme.cl).

Los profesionales docentes, a pesar de pertenecer a este mismo organismo municipal, no todos han recibido una misma formación. Esto se debe a los distintos cambios en que nuestro país se ha visto afectado a lo largo de la historia y las diferentes prioridades existentes en cada época.

En el siguiente apartado se describen brevemente las formas con las cuales una persona podía acceder al título de profesor.

FORMACION PROFESIONAL DOCENTE. En nuestro país han existido principalmente tres sistemas de formación de profesores básicos: las escuelas normales (creadas en 1842 y cerradas en 1974), las universidades y, desde 1982, los institutos profesionales, diferenciándose estos últimos sólo por la libertad que tenían de escoger las currículos y la duración de la carrera (Díaz, Larraín y Ugarte 1992).

Las escuelas normales surgen a mediados del siglo XIX con el propósito de preparar profesores que se hicieran cargo de la educación de las clases populares bajo el supuesto de que la educación de las masas permitiría una mayor estabilidad política y un progreso social y económico. Los futuros profesores venían de un estrato popular, 
que no pertenecía al sistema político y social dominante; se les exigía como requisito de ingreso solamente una base moral sólida y conocimientos elementales. El gobierno les asignaba una pensión (que cubría gastos de mantención y vestuario), contrayendo el estudiante la obligación de servir a su egreso durante siete años como preceptor donde fuera asignado. Fue en esta época que se instauró el régimen de internado, que junto con la exigencia de un origen determinado aseguraba, en cierto sentido, la adscripción de los sectores populares al orden social (Cox y Gysling 1990).

La formación de profesores en la Universidad, la cual comenzó con la creación del Instituto Pedagógico de la Universidad de Chile en 1889, tenía una orientación hacia la formación de profesores para la educación secundaria. Así, la formación de profesores básicos en las universidades es un hecho relativamente reciente en la historia del país, ya que durante años la formación universitaria estuvo dirigida principalmente a preparar profesores para desenvolverse en un ámbito secundario y no primario (Cox y Gysling 1990).

Con lo anteriormente expuesto, queda claro que se está frente a un magisterio que ha vivido de distintas maneras su formación profesional, con distintos objetivos y en distintas épocas. Esto permite reflexionar de forma tal, que se podría creer en la posibilidad de que esta diferencia en la formación y adquisición de contenidos del profesor de una u otra manera podrían estar dando las bases para realizar perfiles de docentes que identifiquen claramente grupos reunidos por sus características comunes pero diferenciados entre sí. Es por esto que a continuación se hace referencia a la investigación psicográfica, ya que es ésta la que, a partir de distintas variables, logra la creación de perfiles, tema esencial de nuestro estudio.

INVESTIGACION PSICOGRAFICA. La investigación psicográfica debe sus comienzos a la psicología del consumidor y la segmentación del mercado. Tiene como objetivo indagar a partir de categorías de variables actitudinales, de intereses, de opiniones (conocidas como AIO) y de estilos de vida en diferentes grupos de consumidores. Para tal fin, la investigación de mercados utiliza como herramienta los cuestionarios psicográficos, los cuales se elaboran a partir de enunciados referentes a las categorías anteriormente mencionadas. Además, explora la incidencia de los factores psicológicos, sociológicos y antropológicos bajo un escenario de toma de decisión o inclinación sobre una idea, valor o cosa con resultados que permiten ser representativos de un universo (www.yanhaas.com 2004).

Los perfiles psicográficos se pueden definir mediante la investigación cualitativa de las variables AIO a través de sesiones de grupo donde se hace una primera segmentación por variables demográficas, para posteriormente administrar los cuestionarios psicográficos con los datos obtenidos en la fase anterior. Al analizar cuantitativamente y en profundidad los datos obtenidos podemos encontrar similitudes entre grupos heterogéneos así como diferencias entre grupos homogéneos. Estas diferencias se deben generalmente a los distintos perfiles psicográficos (Klainer 2000).

Como se mencionó anteriormente, los psicográficos son una herramienta para segmentar el mercado, pero para los fines de este estudio las variables serán investigadas sobre la base de qué actividades, intereses, opiniones y estilos de vida de los sujetos los llevaron a ser profesores de educación básica y los mantienen aún en su ejercicio profesional. 
Si se toma en cuenta la posibilidad de que existieran perfiles de profesores agrupados por las variables anteriormente mencionadas, es de real interés saber si éstas tienen algún tipo de relación con las variables del síndrome de Burnout, o si bien es éste el que podría determinar la presencia de los distintos perfiles en cuestión.

De acuerdo a la bibliografía revisada, se encontró un estudio de la Universidad de Córdoba en España (Moriana y Herruzo 2004), el cual da cuenta de la presencia de dicho síndrome en el cuerpo docente y su gran importancia por traer consecuencias no sólo para el profesional, sino que también para el establecimiento educacional, tales como el aumento anual de licencias médicas y psiquiátricas, el incremento del ausentismo laboral, el enorme gasto en sustituciones y el bajo rendimiento en el trabajo (García 1991).

Es por esto que se considera pertinente incluir al presente estudio las variables del síndrome de Burnout en el profesorado ariqueño, razón por la cual a continuación se describe el concepto de dicho síndrome.

SINDROME DE BURNOUT. También conocido como "síndrome de quedarse quemado en el trabajo". La palabra Burnout proviene del verbo inglés "to burn": quemarse, burn out: haberse quemado y forma parte de lo que en Psicología se conoce como "fatiga laboral" o desgaste profesional, conceptualizado así por Maslach y Jackson (1986).

El Burnout ha sido definido de diferentes formas; sin embargo, existiría consenso general en entender el término como "una respuesta a un estrés emocional crónico, cuyos rasgos principales son el agotamiento físico y psicológico, una actitud fría y despersonalizada en la relación con los demás y un sentimiento de inadecuación a las tareas que se han de realizar" (Maslach y Jackson 1981).

En la V Región y a partir de estudios de la Escuela de Psicología de la Universidad Católica de Valparaíso se construyó y propuso la siguiente definición: "Síndrome resultante de un estado prolongado de estrés laboral, derivado de factores macro y microsociales, que afecta a personas cuya profesión implica una relación con otros, en la cual la ayuda y el apoyo ante los problemas es el eje central del trabajo" (Asún 2001).

El estar "quemado" por estrés en el ámbito laboral se mide a través del Inventario Maslach Burnout que básicamente incluye: (a) Agotamiento Emocional, que es la pérdida de atractivo, tedio y disgusto causado por las tareas y el trabajo; disminución del interés y satisfacción laboral. Además, hay sentimientos de fracaso e indefinición por haber intentado modificar su situación sin resultado. (b) Despersonalización, que es un tipo de conducta caracterizado por falta de interés, objetivación burda y somera de los problemas, pérdida de empatía e incluso sentimientos de culpa por la situación vivida. Esta característica se constituye en el núcleo del Burnout y en un mecanismo reactivo como consecuencia del cambio realizado en la fase anterior. (c) Realización Personal, que es el sentimiento de competencia y éxito en el trabajo del profesional, que conlleva a una evaluación positiva por parte del sujeto al sentirse contento consigo mismo y satisfecho con sus resultados laborales.

El síndrome se manifiesta cuando el individuo no es capaz de abordar las situaciones de estrés de forma adaptativa y positiva, a la vez que se siente impedido para modificar la situación problemática. Como consecuencia, acaba utilizando el escape o la evitación como una forma de enfrentamiento al estrés. Al no enfrentarse a las situaciones, los problemas no se resuelven, crecen, dando paso así a un proceso acumulativo de malestar prolongado que acaba por desgastar al profesional, quien finalmente termina sintiéndose indefenso (Vinaccia y Alvarán 2004). 
El Burnout puede afectar a todas las profesiones asistenciales, pero especialmente a la docencia y la asistencia sanitaria, produciendo un deterioro en las relaciones familiares y sociales, pérdida de interés en el trabajo, ausentismo laboral, sentimientos de fracaso y disminución de la autoestima. Así como también este síndrome se relaciona con el insomnio, agotamiento físico y hasta con el uso excesivo de alcohol y drogas (Bruce e Ibaceta 1994).

La diferencia fundamental entre el estrés simple y el síndrome de Burnout es que, mientras que el estrés puede desaparecer tras un periodo adecuado de descanso, el Burnout no declina con las vacaciones ni con otras formas de reposo (Asún 2001).

Ya definidos los principales conceptos referidos a la presente investigación, se procede a presentar paso a paso el método utilizado en esta misma, describiendo a los participantes que conformaron la muestra, los instrumentos utilizados y el procedimiento, tanto cualitativo como cuantitativo, para la construcción y validación de dicho Inventario.

\section{METODO}

Participantes. Se realizó un muestreo probabilístico por conglomerado multietápico, para lograr una muestra lo más representativa de las escuelas municipales de la ciudad de Arica. Así la muestra quedó compuesta por nueve establecimientos educacionales: Escuelas G-27, D-11, D-4, D-14, D-10, E-1, D-21, D-16 y colegio integrado, de las cuales 99 profesores de educación básica fueron elegidos aleatoriamente para participar en el estudio. De los participantes 73 correspondían a mujeres y 26 a hombres. Y del total de éstos, un $79 \%$ poseía más de 21 años ejerciendo como profesor.

Instrumentos. Se realizaron entrevistas en profundidad a los profesores para acceder a sus historias de vida y obtener información necesaria que permitiera crear un instrumento de evaluación. Se utilizó la técnica de Grupos Focales para la discusión, con el fin de conocer su realidad desde su propio marco de referencia.

Se empleó el cuestionario Psicográfico construido a partir de las entrevistas individuales y los grupos focales realizados, agregando el Inventario de Maslach Burnout (MBI), desarrollado por Maslach y Jackson (1986). Este instrumento supone el Burnout como un constructo tridimensional midiendo Cansancio Emocional, Despersonalización y Realización Personal. Se trata de un Inventario autoadministrable que posee una consistencia interna y una fiabilidad cercana al 0,9 y está constituido por 22 ítemes en forma de afirmaciones.

Procedimiento. Se realizó un primer contacto con los directores de las escuelas municipales de distintos sectores de la ciudad escogidos al azar, y con una previa autorización del Director del SERME se consiguieron las citas con los profesores para realizar las entrevistas en profundidad individuales y grupales.

Las entrevistas individuales se realizaron a dos profesores de cada escuela seleccionada $\mathrm{y}$, utilizando un método de entrevista semidirigida, las entrevistadoras iban completando un formato de preguntas previamente escrito. El objetivo de dichas entrevistas era realizar una aproximación temática de la vida laboral y familiar del profesor, sus inquietudes, intereses y motivaciones personales para luego poder crear el instrumento psicográfico. 
Con la información obtenida y ya con algunas directrices temáticas, se procedió a realizar cuatro grupos focales para conocer en forma generalizada la realidad de los docentes según su propio marco de referencia. Los grupos estuvieron compuestos por 12 profesores escogidos al azar por el director de cada establecimiento municipal y las sesiones tenían una duración aproximada de 60 minutos.

Cabe destacar que los profesores que participaron en las entrevistas individuales y en las grupales forman parte de la muestra total $(n=99)$.

La construcción del Inventario Psicográfico para Profesores Básicos (IPPB) adquirió el formato de escala de Likert basándose en la información obtenida de las entrevistas individuales y grupales previamente realizadas. El procedimiento fue el siguiente:

1. Identificación de las dimensiones: basadas en las variables consideradas por el AIO, se determinaron las dimensiones de evaluación que debe contemplar el instrumento, con el fin de especificar las temáticas relevantes que permitirán distinguir las características de los docentes.

2. Construcción de ítemes: se confeccionaron 231 ítemes, esperando que el instrumento en su versión final constara con a lo menos un tercio de éstos.

3. Validez de contenido: la composición de la prueba se obtuvo luego de someter los 231 ítemes a un estudio de validez de contenido a través de un criterio de jueces. Los reactivos fueron evaluados por tres psicólogos expertos del área de la metodología de la Universidad de Tarapacá. Los criterios utilizados fueron redacción, comprensión y contenido de los ítemes, para lo cual se facilitó a los jueces la definición operacional de cada escala.

Bastaba con que uno de los tres jueces señalara que un reactivo no era adecuado para que éste fuese eliminado. Así, las dimensiones obtenidas a través de este procedimiento fueron: Comienzos, Estilo de vida, Miedos, Dedicación y Valores.

Finalmente el Inventario quedó con 140 reactivos.

Se incluyó el Inventario de Maslach Burnout para ver si correlacionaba con algunos de los factores o dimensiones ya presentados.

4. Aplicación de la prueba: una vez eliminados los ítemes de acuerdo al criterio de jueces expertos, aplicamos la prueba constituida por los 140 reactivos a la muestra de 99 profesores.

5. Análisis de constructo: se realizó el análisis de los datos a través del Paquete Estadístico para Ciencias Sociales (SPSS), versión 10.0 para Windows, realizando en una primera instancia un Análisis Factorial Exploratorio de componentes principales con rotación Varimax con el total de los ítemes que constituyen la prueba, para luego determinar la confiabilidad interna del instrumento a través del procedimiento de Alfa de Cronbach.

6. Análisis de conglomerados y discriminante: una vez determinados los puntajes obtenidos por los sujetos en cada factor evaluado por el instrumento (mediante suma simple de los reactivos que conforman cada factor) se procedió a realizar un análisis de cluster con el procedimiento " $\mathrm{K}$ mean cluster" previamente determinando cinco perfiles, los cuales fueron posteriormente confirmados mediante el Análisis Discriminante. 


\section{RESULTADOS}

El Análisis Factorial agrupó a los ítemes de la prueba en cinco factores a partir de los cuales se estimó el coeficiente de confiabilidad de cada uno de ellos (ver tabla 1). El coeficiente de confiabilidad para el total de los ítemes fue de alfa 0,93.

Tabla 1

Confiabilidad y porcentaje de varianza por factor

\begin{tabular}{|l|c|c|}
\hline \multicolumn{1}{|c|}{ Factor } & $\begin{array}{c}\text { Coeficiente Alfa de } \\
\text { Conbrach }\end{array}$ & \% Varianza \\
\hline Estilo de vida & 0,92 & 32,48 \\
\hline Miedos & 0,87 & 30,89 \\
\hline Dedicación & 0,88 & 33,53 \\
\hline Valores & 0,83 & 30,39 \\
\hline Comienzos & 0,83 & 34,8 \\
\hline
\end{tabular}

Por lo tanto, se mantuvieron cinco factores agrupados por el análisis descrito anteriormente, los cuales pueden ser redefinidos de la siguiente manera:

Factor 1 "Estilo de vida": Son las actividades, intereses y opiniones de los profesores referentes a su quehacer profesional y su vida cotidiana.

Factor 2 "Miedo": Se refiere a las ansiedades, temores e inhibiciones que el profesor percibe como amenaza tanto en su vida cotidiana, su ejercicio profesional y su futuro retiro laboral, poniéndolo en una posición inestable y vulnerable.

Factor 3 "Dedicación": Hace referencia a la disposición, tiempo y constancia que el docente invierte para el logro de sus metas profesionales.

Factor 4 "Valores": Son las creencias o convicciones que posee el docente y que entrega o traspasa a sus alumnos como parte del proceso de aprendizaje, siendo éste su interés principal más allá de la entrega de conocimientos, transformando su rol de profesor en uno de formador.

Factor 5 "Comienzos": Refiere a todas aquellas actividades, intereses y opiniones relacionadas a la infancia y vida estudiantil del profesor que motivaron su preferencia por el ejercicio docente.

De esta forma, la prueba final corresponde a un instrumento de lápiz y papel que consta de un total de 99 ítemes (ver tabla 2) estructurados como escala tipo Likert, frente a los que la persona debía responder entre cinco alternativas, que iban desde la afirmación muy en desacuerdo hasta тиy de acuerdo. Adicionalmente, contempló un breve cuestionario demográfico de 17 preguntas, correspondientes a identificar sexo, edad, estado civil, número de hijos, ciclo de enseñanza en el que ejerce, años de ejercicio docente, establecimiento educacional, entre otras, y por último, se adjuntó el Inventario Maslach Burnout. 
Posteriormente, se realizó el Análisis de Conglomerados con el fin de agrupar a los profesores en función de la similitud existente entre ellos y así poder identificar los distintos perfiles (ver tabla 2).

\section{Tabla 2}

Promedios de las variables por conglomerados

\begin{tabular}{|l|r|r|r|r|r|}
\hline & \multicolumn{5}{|c|}{ Conglomerado } \\
\cline { 2 - 6 } & \multicolumn{1}{|c|}{$\mathrm{C} 1$} & \multicolumn{1}{c|}{$\mathrm{C} 2$} & \multicolumn{1}{c|}{$\mathrm{C} 3$} & \multicolumn{1}{c|}{$\mathrm{C} 4$} & \multicolumn{1}{c|}{ C5 } \\
\hline Estilo de vida & 3,43 & 4,56 & 4,29 & 3,55 & 3,82 \\
\hline Miedos & 2,51 & 2,29 & 3,55 & 1,94 & 2,60 \\
\hline Dedicación & 3,34 & 4,34 & 4,10 & 2,52 & 3,66 \\
\hline Valores & 4,10 & 4,77 & 4,64 & 4,47 & 4,38 \\
\hline Comienzos & 2,76 & 4,40 & 4,31 & 4,29 & 4,15 \\
\hline Total Casos & 8 & 26 & 31 & 5 & 29 \\
\hline
\end{tabular}

Finalmente, se confirmaron estos conglomerados mediante el Análisis Discriminante, el cual arrojó una correlación canónica de 0,89, altamente significativa. Es así que a continuación se describen cualitativamente los perfiles de acuerdo a los resultados obtenidos en la tabla 2.

$C l=$ "Indiferentes": son sujetos que llegaron al quehacer docente debido al azar, ya que ni en su infancia ni vida estudiantil existieron modelos significativos que motivaran la elección de esta profesión. Para ellos, el ser profesor es una actividad más que les permite ganarse la vida. Sus actividades, intereses y opiniones no hacen referencia a su quehacer profesional. No invierten mayor tiempo, disposición ni constancia para el logro de sus metas profesionales. Si bien, en su vida cotidiana, en su ejercicio profesional y en su futuro retiro laboral se les presentan ansiedades y temores, éstos no son percibidos como amenazas que los ubiquen en una posición de inestabilidad.

$C 2=$ "Realizados": estos sujetos se caracterizan porque desde su infancia contaron con apoyos y/o modelos significativos para dedicarse a la docencia. Es por esto que su vida cotidiana está ligada fuertemente a su quehacer profesional, es decir, son profesores las 24 horas del día, ya que sus conversaciones, intereses y acciones giran en torno a su medio laboral, invirtiendo mayor tiempo, dedicación y constancia para el logro de sus metas profesionales. Se destaca su interés principal, más allá de la entrega de conocimientos, el traspasar valores a sus alumnos, transformando su rol de profesor en uno de formador. 
C3= "Dedicados Temerosos": estos sujetos manifestaron desde su infancia el interés por el ejercicio docente, manteniéndose éste en la actualidad, el cual se manifiesta a través de sus actividades e intereses que hacen referencia a su quehacer profesional. Dedican tiempo y constancia al logro de sus metas laborales, las cuales incluyen tanto la entrega de conocimiento como de valores a sus alumnos. Se caracterizan por presentar ansiedades y temores percibidos como amenaza en su vida cotidiana y profesional, poniéndolos en una posición de inestabilidad y vulnerabilidad.

C4= "Triviales": son sujetos que llegaron a ser profesores por una elección motivada en modelos significativos relacionados a la docencia durante su infancia. Sin embargo, para ellos el ser profesor es una actividad más dentro de su vida, sólo cumplen su rol profesional dentro de la sala de clases y horario de trabajo, no invirtiendo mayor tiempo ni constancia más allá de lo necesario. Sus valores, creencias y convicciones que traspasan a sus alumnos son considerados como parte del proceso de aprendizaje. Se caracterizan por no presentar temores e inhibiciones que dificulten su ejercicio profesional ni su futuro retiro laboral.

C5= "Desilusionados": este grupo ha visto favorecida su incorporación en la docencia con apoyos y modelos para dedicarse a esta actividad profesionalmente, sin embargo, el tiempo y la disposición a dicha actividad no va más allá de lo requerido. Son personas que no conjugan su vida laboral con su vida cotidiana. Se destacan por percibir muchas barreras y temores para el desempeño de su carrera profesional.

Una vez identificados los perfiles, se relacionaron éstos con las variables del Burnout a través del Análisis ANOVA (prueba F y el Test Post Doc Duncan), con el objetivo de realizar un análisis más acabado de éstos (ver tabla 3). Los resultados para la variable Cansancio Emocional fue de una $\mathrm{F}=4,136$ con un nivel de significancia de .004, para la variable Despersonalización una $\mathrm{F}=4,829$ y un nivel de significancia de .001 y para la variable Realización Personal una F= 2,991 y un nivel de significancia de .023.

\section{Tabla 3}

Análisis comparativo por conglomerado de las subescalas del Inventario de Maslasch.

\begin{tabular}{|c|c|c|c|c|}
\hline Conglomerados & $\mathrm{N}$ & $\begin{array}{c}\text { Cansancio } \\
\text { Emocional }\end{array}$ & Despersonalización & $\begin{array}{c}\text { Realización } \\
\text { Personal }\end{array}$ \\
\hline C1: Indiferentes & 7 & 11.57 & 5.29 & 40.00 \\
\hline C2: Realizados & 25 & 11.36 & 1.68 & 45.24 \\
\hline C3: Dedicados Temerosos & 27 & 21.89 & 6.93 & 40.37 \\
\hline C4: Triviales & 4 & 10.50 & 2.00 & 37.50 \\
\hline C5: Desilusionados & 29 & 13.52 & 2.93 & 40.69 \\
\hline Sig. & & .081 & .076 & .096 \\
\hline
\end{tabular}


De acuerdo a la tabla 3, se puede observar que el C3, compuesto por los profesores "Dedicados Temerosos", presenta un elevado Cansancio Emocional en relación a los demás conglomerados, con un promedio de 21.89 puntos obtenidos en esta variable perteneciente al Inventario de Maslasch. A su vez, el C4, el cual agrupa a los profesores "Triviales", es el que presenta menor cansancio. Con respecto a la Despersonalización, los profesores "Dedicados Temerosos" (C3) se encuentran altamente despersonalizados con un promedio de 6.93, seguido por el C1 de los "Indiferentes" con un promedio de M=5.29. A diferencia del C5 de los "Desilusionados" que presenta el menor promedio ( $M=2.93)$. Con respecto al promedio de la subescala Realización Personal, es relevante destacar que

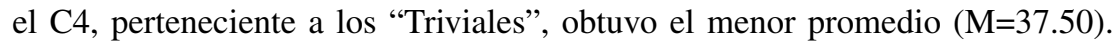

A partir de estos resultados, se realizan las discusiones y conclusiones de la investigación, que a continuación se presentan.

\section{DISCUSION Y CONCLUSIONES}

Al explorar la incidencia de los factores anteriormente descritos (estilo de vida, miedos, dedicación, valores y comienzos) sobre la forma de vida de los profesores, se encontró que éstos se agrupan en cinco conglomerados (Indiferentes, Realizados, Dedicados Temerosos, Triviales y Desilusionados) de los cuales se han podido visualizar dos grandes perfiles descritos a continuación:

$P P 1=$ "Perfil Optimo": compuesto por aquellos sujetos Realizados (C2) y los Dedicados Temerosos (C3) que se caracterizan por encontrarse motivados a ejercer la docencia desde su infancia y han dedicado su vida al logro de este objetivo, invirtiendo tiempo, dedicación y constancia a su trabajo más allá de lo requerido. La única diferencia entre ambos conglomerados radica en el monto de temor que sienten con respecto a su estabilidad laboral y futuro retiro profesional.

$P P 2=$ "Perfil Desalentado": conformado por los profesores Indiferentes (C1), los Triviales (C4) y los Desilusionados (C5). Este grupo cumple su rol profesional sólo en las horas de trabajo, no invirtiendo mayor tiempo ni dedicación para el logro de sus metas laborales. Si bien los profesores Triviales y los Desilusionados alguna vez estuvieron motivados al inicio de sus carreras, éstos fueron perdiendo con el tiempo el interés por el ejercicio de su profesión. A diferencia de los Indiferentes que llegaron a ser profesores no por vocación sino por el azar, no se sienten motivados por su trabajo y, por lo tanto, no le dedican mayor tiempo ni esfuerzo a éste.

Por otro lado, con respecto al síndrome de Burnout, contrario a lo que se esperaba, los profesores no presentaron puntuaciones que hagan suponer que lo padecen. Sin embargo, si se realiza un análisis más acabado de cada uno de los perfiles y sus conglomerados que los conforman, relacionándolos con las variables de dicho síndrome y la información obtenida en las entrevistas individuales y grupos focales, se podría decir que: A pesar de las dificultades del medio, percibidas por los profesores como los bajos recursos destinados por el SERME, el bajo compromiso de los apoderados, la elevada carga horaria y las nuevas propuestas de la Reforma Educacional, entre otras, el 58\% de la muestra correspondiente al Perfil Optimo ha mantenido su vocación a través del tiempo, ya que 
ha visto resultados tangibles a través de su ejercicio profesional acordes a los objetivos de la educación básica como lo son la lectoescritura, el manejo de las funciones básicas de las matemáticas y la comprensión general del medio y su entorno social, lo cual podría estar relacionado a la alta puntuación obtenida en la escala de Realización Personal y la ausencia de síndrome de Burnout.

Es interesante observar también que dentro de este perfil existe una diferencia entre los dos conglomerados que lo conforman, los Realizados y los Dedicados Temerosos, caracterizando a estos últimos como profesores que perciben mayores amenazas tanto en su vida cotidiana, su ejercicio profesional y su futuro retiro laboral. Si dicho temor se relacionara con la alta despersonalización que presentan, podríamos decir que ésta es una respuesta ante la vulnerabilidad percibida, provocando sentimientos de inseguridad y frustración, y como consecuencia de esto un alto Cansancio Emocional.

Ahora bien, estos factores estresantes que están afectando sólo a los Dedicados Temerosos podrían ser, y de acuerdo a lo conversado durante las entrevistas individuales y grupales, la situación que actualmente está viviendo el magisterio con respecto a la nueva Reforma Educacional, que incluye la Evaluación Docente propuesta por el Ministerio de Educación, así como también la imposibilidad de acceder a una jubilación suficiente como para solventar sus gastos mínimos para vivir durante su vejez. Por lo tanto, se podría decir que los Dedicados Temerosos se sienten más afectados porque están comprometidos con su labor docente, sin tener el reconocimiento social ni el apoyo institucional esperado. A diferencia de los Realizados que no se ven afectados porque podrían estar actuando en ellos, como un factor protector, sus sentimientos de competencia y éxito profesional acompañado de una evaluación positiva de sí mismos que los lleva a estar contentos y satisfechos con sus resultados laborales y personales, siendo la escuela y sus alumnos un motivo de orgullo y realización en sus vidas.

A la luz de esta conclusión, se considera pertinente sugerir el entrenamiento al grupo de los Dedicados Temerosos en estrategias de enfrentamiento de estrés para modificar sus respuestas frente a estos estímulos.

Por otra parte, con respecto al Perfil Desalentado, cabe destacar que los profesores Triviales y los Desilusionados partieron de la base de que desde pequeños quisieron ser profesores y estuvieron motivados por modelos significativos para la elección de la docencia, aunque fueron perdiendo este entusiasmo y dedicación a lo largo de su ejercicio profesional. Se supone que esta disminución es una reacción ante las dificultades del medio educacional que ya hemos descrito (evaluaciones docentes, jubilación inadecuada, etc.). Ahora bien, los profesores Triviales presentan bajos niveles de Cansancio Emocional, interés y satisfacción laboral debido a la falta de metas profesionales que se evidencian en la baja Realización Personal, y los profesores Desilusionados presentan altos niveles de cansancio acompañados de sentimientos de fracaso e indefinición por haber intentado modificar su situación, sin resultado alguno. A diferencia de los Indiferentes, que nunca mostraron motivación por la pedagogía y se ven enfrentados a un alto monto de cansancio por el hecho de trabajar con el primer y segundo ciclo básico simultáneamente, reaccionando frente a los factores estresantes con desinterés y pérdida de empatía, característicos de la Despersonalización. Esto sugiere que dicho perfil se encuentra con un desinterés por su trabajo y por sus relaciones sociales dentro de este mismo, que afectan en la calidad de enseñanza otorgada y en la formación de sus alumnos. 
Así finaliza esta investigación, que ha aportado una visión más general de la docencia acercándose más a la definición del estilo de vida del profesor en base a sus características psicográficas como complemento de las evaluaciones ya existentes, lo cual puede utilizarse como base para una intervención psicológica en vías de mejorar su desempeño y así optimizar la educación básica municipal. Así, se espera haber contribuido con un instrumento que facilite la toma de decisiones por las organizaciones pertinentes, de manera satisfactoria, para la generación de nuevos estudios y/o el perfeccionamiento del IPPB.

Se considera que este Inventario Psicográfico para Profesores Básicos (IPPB) puede llegar a ser una herramienta importante tanto para el trabajo del psicólogo organizacional como educacional ya que es un instrumento válido y confiable que permite sentar bases para la selección de los profesionales docentes, la implementación de estrategias de capacitación a los profesores y el diagnóstico del clima organizacional dentro de los establecimientos educacionales.

\section{BIBLIOGRAFIA}

Asún D. (2001). Desgaste (Burnout) en Servicios Sociales: El riesgo de trabajar ayudando a los otros. Imágenes en Salud Mental II, monografías de la Sociedad de Salud Mental. Pp. 156-157.

Bruce, J. y F. Ibaceta (1994). Estudio Exploratorio del Síndrome de Burnout, en un Grupo de Funcionarios de Enfermería, sometidos a diferentes tensiones en el trabajo. Memoria para optar al título de Psicólogo, Universidad de Tarapacá, Chile.

Colegio de Profesores de Chile A. G. (2004). Informe Estudio de Salud Laboral de los Profesores en Chile. Directorio Nacional, Departamento de Bienestar, en www.colegiodeprofesores.cl/ nacional/informe\%20salud.pdf.

Cox, C. y J. Gysling (1990). La formación del profesorado en Chile. 1842-1987. Santiago: CIDE.

Cuadra, A. (1999). Manual de Análisis Multivariado: Una introducción intuitiva y conceptual basada en el SPSS. Universidad de Tarapacá, Arica, Chile.

Díaz, R., R. Larraín R. y N. Ugarte (1992). La educación y la formación desde una perspectiva sociológica. En J. Ruiz (ed) Formación de Profesores, pp. 123-144. Santiago: CPU.

Estudios psicográficos (2004). www.yanhaas.com

García, M. (1991). Bajas por enfermedad: Una investigación crítica. Cuadernos de Pedagogía $\mathrm{N}^{\circ}$ 251: 80-83. Citado en Moriana, J. A. y Herruzo J. (2004): Estrés y Burnout en Profesores. International Journal of Clinical and Health Psychology. Vol. 4 (3): 599.

Howard, John A. (1989). El comportamiento del consumidor en la estrategia del marketing. Díaz de Santos.

June G., J. Mohammed \& L. Quamina (2001). Teacher Identity in a era of Educational Reform. University of the West Indies, St. Augustine, Trinidad y Tobago.

Klainer, V. (2000). Segmentación Psicográfica, Conocer al Consumidor. En: http://segmento.itam. mx. Instituto Tecnológico Autónomo de México.

Maslach, C. \& S. Jackson (1981). Maslach Burnout Inventory: Research Edition. Palo Alto. California: Consulting Psychologist Press, citado en Desgaste (Burnout) en Servicios Sociales: El riesgo de trabajar ayudando a los otros. Autor: Domingo Asún Salazar, Psicólogo Social Universidad de Valparaíso. Imágenes en Salud Mental II, monografías de la Sociedad Chilena de Salud Mental. Pp. 156. Año 2001.

Maslach, C. \& S. Jackson (1986). Patterns of Burnout among a national sample of public contact workers. Journal of Health and Human Resources Administration 7: 189-212. 
Ministerio de Educación de Chile, MINEDUC (2003). Marco de la Buena Enseñanza. En: www. mineduc.cl

Moriana, J. A. y J. Herruzo (2004). Estrés y Burnout en Profesores. International Journal of Clinical and Health. Vol. 4 (3): 597-621. Universidad de Córdoba, España. En: www.aepc.es/ ijchp/articulos_pdf/ijchp-126.pdf

Pulido, C. y H. Riffo (2004). Construcción de perfiles psicográficos de futbolistas profesionales de Chile. Memoria para optar al título de Psicólogo, Universidad de Tarapacá, Arica, Chile.

Rojas, P. (2003). Validación del emprendimiento a partir de la construcción de un perfil psicográfico en la ciudad de Arica. Memoria para optar al título de Psicólogo, Universidad de Tarapacá, Arica, Chile.

Servicio Municipal: www.serme.cl

Truffello, I. (1999). Sistema Escolar y Educación Pública. Revista Enfoques Educacionales. Vol. 2, $\mathrm{N}^{\circ}$ 2. Departamento de Educación, Facultad de Ciencias Sociales, Universidad de Chile.

Vinaccia, S. y L. Alvarán (2004). El Síndrome de Burnout en una muestra de auxiliares de enfermería: Un estudio exploratorio. Revista Terapia Psicológica de la Sociedad Chilena de Psicología Clínica. Vol. 22 (1): 10-11. 